\title{
System for removing shell pieces from hazelnut kernels using impact vibration analysis
}

\author{
A. Enis Cetin ${ }^{\text {a }}$, Tom C. Pearson ${ }^{\text {b }}$, R. Akin Sevimli ${ }^{\text {a,* }}$ \\ a Electrical and Electronics Engineering Department, Bilkent University, Ankara 06800, Turkey \\ ${ }^{\mathrm{b}}$ USDA-ARS-CGAHR, 1515 College Avenue, Manhattan, KS 66502, USA
}

\section{A R T I C L E I N F O}

\section{Article history:}

Received 13 August 2013

Received in revised form 8 November 2013

Accepted 22 November 2013

\section{Keywords:}

Impact vibration analysis

Line spectral frequencies

Mel-cepstral feature

Hazelnuts

\begin{abstract}
A B S T R A C T
A system for removing shell pieces from hazelnut kernels using impact vibration analysis was developed in which nuts are dropped onto a steel plate and the vibration signals are captured and analyzed. The mel-cepstral feature parameters, line spectral frequency values, and Fourier-domain Lebesgue features were extracted from the vibration signals. The best experimental results were obtained using the melcepstral feature parameters. The feature parameters were classified using a support vector machine (SVM), which was trained a priori using a manually classified dataset. An average recognition rate of 98.2\% was achieved. An important feature of the method is that it is easily trainable, enabling it to be applicable to other nuts, including walnuts and pistachio nuts. In addition, the system can be implemented in real time.
\end{abstract}

(c) 2013 Elsevier B.V. All rights reserved.

\section{Introduction}

Dried nuts are commonly used in processed food industry. The presence of shell fragments and pieces inside foods containing dried nuts are undesirable and can pose a safety concern. In this article, a system for removing shell pieces from hazelnut kernels using impact vibration analysis is described.

Hazelnuts (Fig. 1) are softer and less dense than the shell pieces, so vibration signals, or acoustic emissions, produced by the moment of the impact with a steel plate are different between hazelnuts and the shell pieces, as shown in Fig. 2. As a result, it is possible to design a system for removing shell fragments and pieces from hazelnuts by analyzing the impact signals. Impact sound and vibration analysis systems have been widely used in practice Haff and Pearson (2007), Yorulmaz et al. (2012, 2011), Pearson et al. (2007b), Cetin et al. (2004), Onaran et al. (2005), Pearson et al. (2005, 2007a), Cataltepe et al. (2005, 2004b,a), Cetin et al. (2005), Ince et al. (2008), Buerano et al. (2012), Omid et al. (2010), Chen et al. (2011) and Michihiro and Takahisa (2012). Haff and Pearson developed a sorting system to separate pistachio kernels from in-shell nuts using vibration analysis of a small steel plate after a shell or nut piece impacted it Haff and Pearson (2007). For this system, at the lowest throughput rate, classification accuracies were $96 \%$ for in-shell nuts and $99 \%$ for kernels. For throughput rates between 10 and 40 nuts/s, correct classification ranged from $84 \%$ to $90 \%$ for in-shell nuts. For kernels, the accuracy was

\footnotetext{
* Corresponding author. Mobile: +90 312290 1477; fax: +90 3122664192.

E-mail address: sevimli@ee.bilkent.edu.tr (R. Akin Sevimli).
}

$95 \%$ at 10 and 20 nuts/s and $89 \%$ at 40 nuts/s. The authors based the classification on the cumulative histogram of the signal gradients and did not use the other feature extraction methods, such as those used in acoustic signal processing.

Hazelnut kernels do not stick to the shell in dried hazelnuts. When a hazelnut is cracked the kernel comes out as a separate entity. However large shell pieces may cause problems. They cannot be sieved because some shell pieces may be as large as a kernel. In this article a vibration acoustics based system that is capable of separating large shell pieces from kernels is described.

\section{Materials and methods}

\subsection{Description of system hardware and experiment}

A vibratory feeder (FT00, FMC Corp. Homer City, PA) forces the hazelnuts in a single file from a hopper onto an $90-\mathrm{cm}$-long slide made from stainless steel sheet metal. The slide, inclined at $60^{\circ}$ above the horizontal, terminated above a steel plate, onto which the hazelnuts impacted. The steel plate has dimensions of $8 \times 8 \times 2 \mathrm{~cm}$. Schematic of sorting system is shown in Fig. 3 . A vibration sensor (GS-20DX, Geophone, Geospace Technologies) is mounted onto the steel plate. A laser is used to detect the sliding hazelnuts in the system. When the shell fragments of the hazelnuts pass from the end of the chute, the laser light is blocked, initiating data collection from the vibration sensor. The analog signal is digitized by using chipKIT Uno32. The sensor signal is sampled at $4 \mathrm{KHz}$ for 2048 samples. 


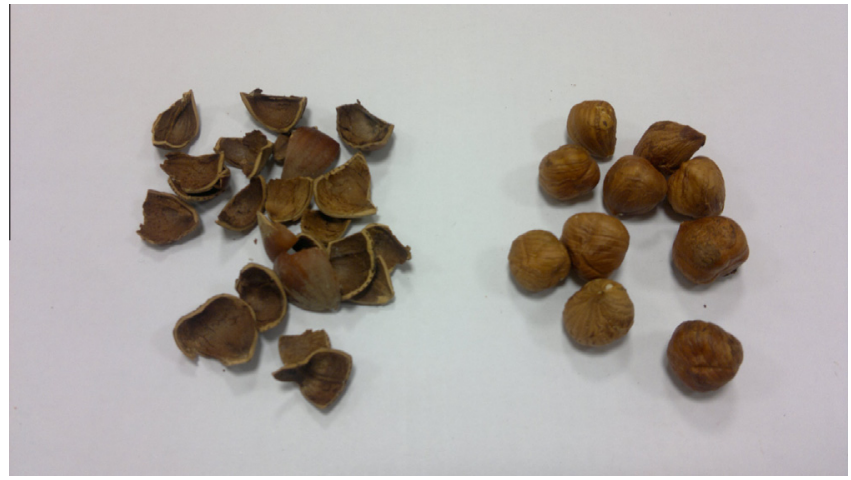

Fig. 1. Hazelnut and shell fragments.

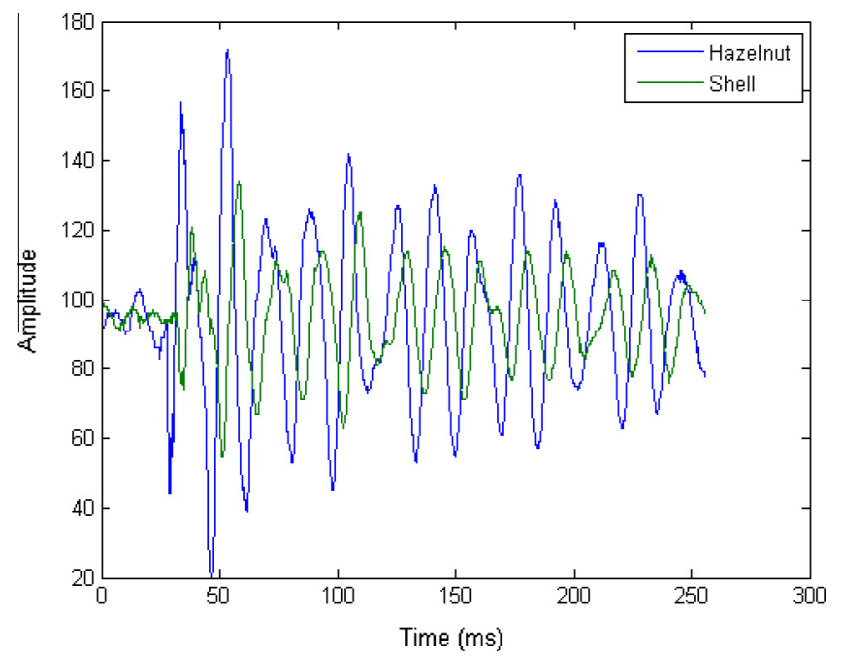

Fig. 2. Vibration signal example for hazelnuts and shell pieces.

Vibration impact signals were collected for 130 hazelnuts and 152 shell pieces from the Akcakoca region of Turkey. After data collection, the vibration signals are processed off-line. The average of the sampled signals is removed by mean-centering before calculating the feature vectors. The cumulative histogram of gradients used by Pearson and Haff, the mel-cepstrum, the line spectrum frequencies, the frequency amplitude bins, and the Lebesgue features are computed as described in Sections 2.1, 2.2, 2.3, 2.4, 2.5 and 2.6, respectively. After all of the features are extracted, classification is

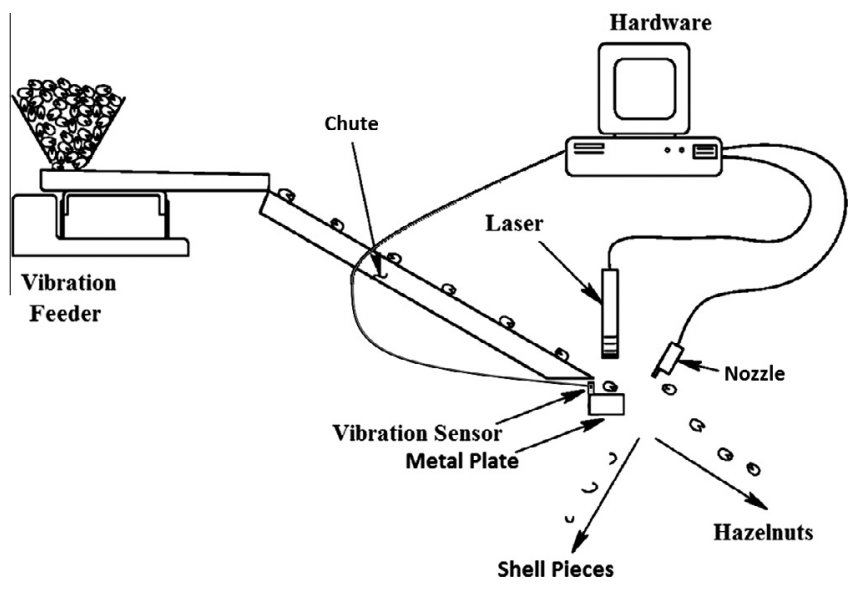

Fig. 3. Schematic of sorting system. performed using a support vector machine (SVM), as described in Section 2.7.

\subsection{Cumulative histogram of gradients (CHOG)}

Haff and Pearson (2007) used cumulative histogram of gradients as a feature vector to separate pistachio kernels from in-shell nuts.

Let $x[n]$ be the zero mean vibration signal and $x_{m}$ represent a normalized version of $x$, calculated as follows:

$x_{m}[n]=x[n] / \max (|x|)$

Then, the gradient $g[n]$ is calculated as follows:

$g[n]=\left|x_{m}[n-1]-x_{m}[n+1]\right|$

Then, a histogram of this gradient signal is calculated. In Haff and Pearson (2007), the authors observed that most of these gradient signals do not contain any values larger than 0.5 ; to reduce the number of bins of the histogram, they clamped gradient signals with a threshold of 0.5 as follows:

$g_{c}[n]=\min (g[n], 0.5)$

Then, the histogram $h[n]$ of $g_{c}[n]$ is calculated. The bins of this histogram covers the range from 0 to 0.5 . Finally, a cumulative histogram of gradients $b[k]$ is calculated as follows:

$b[k]=\sum_{j=0}^{k} h[j], \quad k=0,1, \ldots, K$

where $K$ is the number of feature parameters. The vector $b[k]$ is used to train a SVM with two classes. The first class consists of the vibration signals of the shell pieces, and the second class contains the hazelnut signals.

\subsection{Mel-Cepstrum}

The mel-cepstrum or mel-frequency cepstral coefficient (MFCC) vector is the most widely used feature vector in speech and sound recognition. Cetin et al. (2004) also used the mel-cepstrum to classify the impact sounds of open and closed shell pistachio nuts. Let $X[k]$ represent the $N$-point discrete Fourier transform (DFT) of $x[k]$.

$X[k]=\sum_{n=0}^{N-1} x[n] e^{-j \frac{2 \pi k n}{N}}, \quad k=0,1, \ldots, N-1$

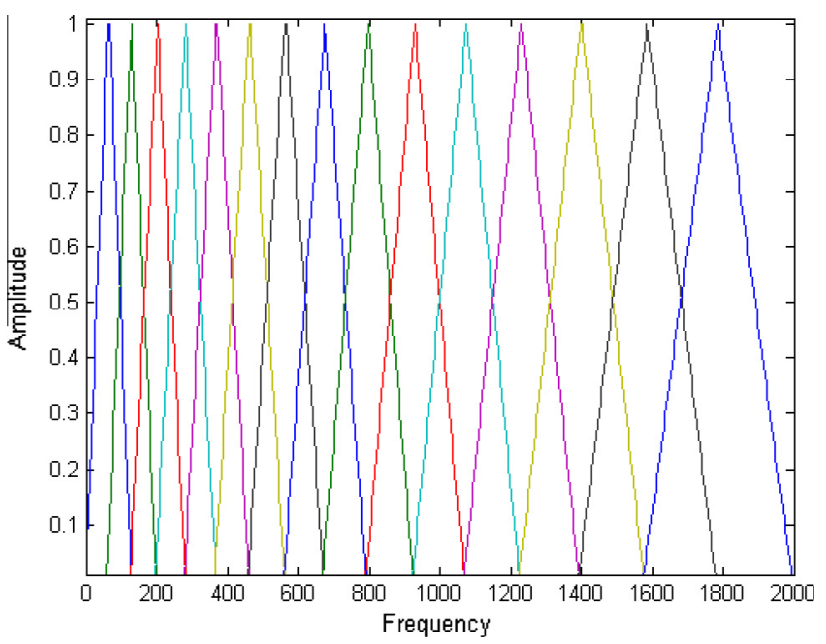

Fig. 4. Filter Bank with Mel-scale. 
Table 1

Frequency boundaries of mel-cepstral filters $(R=15)$.

\begin{tabular}{ccc}
\hline Filter ID & Lower bound $(\mathrm{hz})$ & Upper bound $(\mathrm{hz})$ \\
\hline 1 & 0 & 130 \\
2 & 63 & 203 \\
3 & 130 & 282 \\
4 & 203 & 368 \\
5 & 282 & 462 \\
6 & 368 & 564 \\
7 & 462 & 676 \\
8 & 564 & 796 \\
9 & 676 & 928 \\
10 & 796 & 1071 \\
11 & 928 & 1227 \\
12 & 1071 & 1397 \\
13 & 1227 & 1581 \\
14 & 1397 & 1782 \\
15 & 1581 & 2000 \\
\hline
\end{tabular}

Table 2

Frequency boundaries of mel-cepstral filters $(R=20)$.

\begin{tabular}{rrr}
\hline Filter ID & Lower bound $(\mathrm{hz})$ & Upper bound $(\mathrm{hz})$ \\
\hline 1 & 0 & 97 \\
2 & 47 & 150 \\
3 & 97 & 206 \\
4 & 150 & 266 \\
5 & 206 & 330 \\
6 & 266 & 399 \\
7 & 330 & 472 \\
8 & 399 & 549 \\
9 & 472 & 632 \\
10 & 549 & 720 \\
11 & 632 & 815 \\
12 & 720 & 915 \\
13 & 815 & 1022 \\
14 & 915 & 1136 \\
15 & 1022 & 1258 \\
16 & 1136 & 1388 \\
17 & 1258 & 1527 \\
18 & 1388 & 1674 \\
19 & 1527 & 1832 \\
20 & 1674 & 2000 \\
\hline
\end{tabular}

The DFT is weighted using a bank of filters, as shown in Fig. 4, where the filter bandwidth increases with frequency. The Fourier-domain filter outputs are calculated as follows:

$E_{\text {mel }}(l)=\frac{1}{A_{l}} \sum_{k=L_{l}}^{U_{l}}\left|V_{l}[k] X[k]\right|^{2}, \quad l=0,1, \ldots, R-1$

where $L_{l}$ and $U_{l}$ are the frequency band edge values of the $l$ th filter. In this article, several MFCC vectors were computed using $R=15$, 20,30 , and 100 band filter banks. The frequency boundaries for 15 and 20 band filters are given in Tables 1 and 2, respectively. $A_{l}$ is the normalization factor:

$A_{l}=\sum_{k=L_{l}}^{U_{l}}\left|V_{l}[k]\right|^{2}, \quad l=0,1, \ldots, R-1$

The filter output, $E_{m e l}$ for each band is calculated as follows:

$E_{\text {mel }}(l)=\frac{1}{A_{l}} \sum_{k=L_{l}}^{U_{l}} V_{l}[k]|X[k]|, \quad l=0,1, \ldots, R-1$

Finally, the MFCC vector coefficients are calculated as follows;

$c[m]=\frac{1}{R} \sum_{l=0}^{R-1} \log \left(E_{m e l}(l)\right) \cos \left(\frac{2 \pi}{R} l m\right), \quad m=0,1, \ldots, R-1$ where $R$ is the number of weighted filters in the above equation. There are 15, 20, 30, 50 and 100 weighted filters in our tests. The purpose of using discrete cosine transform (DCT) in Eq. (9) is to decrease the correlation between the filter energy output coefficients. The MFCC coefficients decay as the index parameter $m$ increases. Therefore, it may not be necessary to compute all of the $R$ coefficients. MFCC $(L, R)$ indicates that the first $L$ out of $R$ coefficients are used in the classification.

As indicated in Section 3, the best experimental results are obtained using the MFCC coefficients.

\subsection{Line spectrum frequency (LSF) Coefficients}

We compared the MFCC feature parameters to various other feature parameters used in speech and signal processing. The LSF parameters are one of the sound feature vectors used in speech recognition and analysis (Erzin et al., 1995). In this study, LSFs are also used to extract features from the vibration signals.

Let $A(z)=\sum_{k=1}^{p} a_{k} z^{-k}$ be the polynomial that is extracted from the vibration sensor signals by linear predictive coding (LPC) (Rabiner and Schafer, 1979). From this polynomial, two polynomials of degree $p+1$ are generated:

$P(z)=A(z)+z^{-(p+1)} A\left(z^{-1}\right)$

$Q(z)=A(z)-z^{-(p+1)} A\left(z^{-1}\right)$

The LSF parameters $w_{i}$ are defined as roots of polynomials $P(z)$ and $Q(z)$. The LSF parameters are located on the unit circle, and they approach each other whenever the signal spectrum has high values. Therefore, the LSF parameters represent the shape of the spectrum. In Fig. 5, a vibration signal spectrum and the corresponding LSF parameters are shown. In speech processing, 10-20 LSF parameters are used. In our case, the best results are obtained for 15 LSFs. However, the results indicate that the LSF representation is inferior to the MFCC representation discussed in Section 2.3.

\subsection{Frequency amplitude bins (FAB)}

We also attempted to represent the vibration signals using Fourier domain information.

Fourier transform coefficients and subband energies were used to represent the impact vibration signals. The frequency spectrum was divided into bands in a logarithmic manner, as was performed

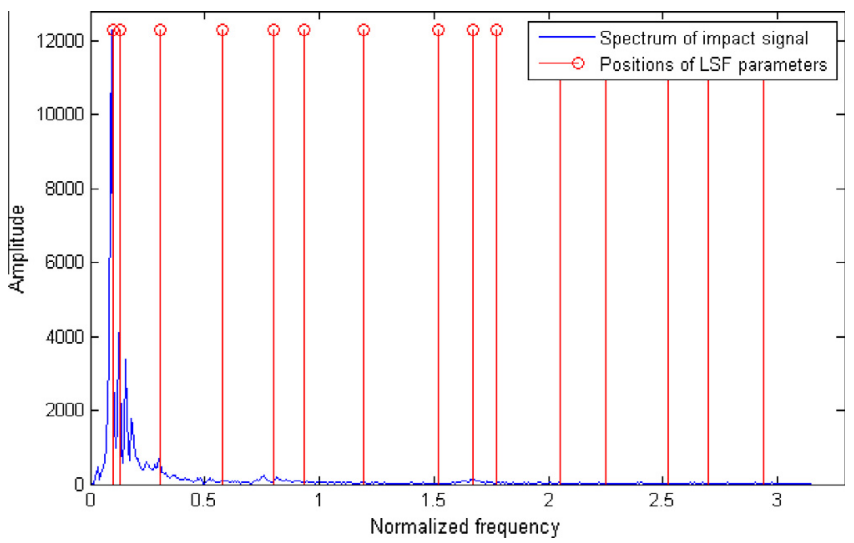

Fig. 5. Spectrum of an average vibration signal and the corresponding LSF parameters. Red lines show LSF parameter's location. (For interpretation of the references to color in this figure legend, the reader is referred to the web version of this article.) 
Table 3

Frequency boundaries of mel-cepstral filters $(R=100)$.

\begin{tabular}{|c|c|c|}
\hline Filter ID & Lower bound (hz) & Upper bound (hz) \\
\hline 1 & 0 & 20 \\
\hline 2 & 10 & 30 \\
\hline 3 & 20 & 39 \\
\hline 4 & 30 & 49 \\
\hline 5 & 39 & 59 \\
\hline 6 & 49 & 70 \\
\hline 7 & 59 & 80 \\
\hline 8 & 70 & 90 \\
\hline 9 & 80 & 101 \\
\hline 10 & 90 & 112 \\
\hline 11 & 101 & 123 \\
\hline 12 & 112 & 134 \\
\hline 13 & 123 & 145 \\
\hline 14 & 134 & 156 \\
\hline 15 & 145 & 168 \\
\hline 16 & 156 & 180 \\
\hline 17 & 168 & 191 \\
\hline 18 & 180 & 203 \\
\hline 19 & 191 & 215 \\
\hline 20 & 203 & 228 \\
\hline 21 & 215 & 240 \\
\hline 22 & 228 & 253 \\
\hline 23 & 240 & 266 \\
\hline 24 & 253 & 279 \\
\hline 25 & 266 & 292 \\
\hline 26 & 279 & 305 \\
\hline 27 & 292 & 319 \\
\hline 28 & 305 & 332 \\
\hline 29 & 319 & 346 \\
\hline 30 & 332 & 360 \\
\hline
\end{tabular}

Table 4

Recognition success rates (sample length is 2048).

\begin{tabular}{|c|c|c|c|}
\hline Feature vector & Shell piece (\%) & Hazelnut (\%) & Average (\%) \\
\hline CHOG (15/15) & 68.0 & 80.8 & 74.4 \\
\hline CHOG $(20 / 20)$ & 74.8 & 85.6 & 80.2 \\
\hline MFCC (15/15/4000) & 97.2 & 98.4 & 97.8 \\
\hline MFCC (20/20/4000) & 96.4 & 99.2 & 97.8 \\
\hline MFCC (10/30/4000) & 95.6 & 99.2 & 97.4 \\
\hline MFCC (15/30/4000) & 96.4 & 99.2 & 97.8 \\
\hline MFCC (10/50/4000) & 95.6 & 99.2 & 97.4 \\
\hline MFCC (15/50/4000) & 96.8 & 98.8 & 97.8 \\
\hline MFCC (10/100/4000) & 96.4 & 99.2 & 97.8 \\
\hline MFCC (15/100/4000) & 97.2 & 99.2 & 98.2 \\
\hline MFCC $(20 / 100 / 4000)$ & 96.8 & 99.2 & 98.0 \\
\hline MFCC (30/100/4000) & 95.2 & 99.2 & 97.2 \\
\hline $\operatorname{LSF}(10 / 15)$ & 86.4 & 100.0 & 93.2 \\
\hline $\operatorname{LSF}(15 / 15)$ & 87.2 & 98.8 & 93.0 \\
\hline $\operatorname{LSF}(15 / 20)$ & 86.8 & 98.4 & 92.6 \\
\hline $\operatorname{LSF}(20 / 20)$ & 87.2 & 98.0 & 92.6 \\
\hline$F A B_{\text {mel }}(15 / 15 / 4000)$ & 96.8 & 93.2 & 95.0 \\
\hline$F A B_{\text {mel }}(20 / 20 / 4000)$ & 96.8 & 93.2 & 95.0 \\
\hline$F A B_{\text {mel }}(10 / 30 / 4000)$ & 97.6 & 95.2 & 96.4 \\
\hline$F A B_{\text {mel }}(15 / 30 / 4000)$ & 95.6 & 95.2 & 95.4 \\
\hline$F A B_{\text {mel }}(10 / 50 / 4000)$ & 98.4 & 96.0 & 97.2 \\
\hline$F A B_{\text {mel }}(15 / 50 / 4000)$ & 97.6 & 95.2 & 96.4 \\
\hline$F A B_{\text {mel }}(10 / 100 / 4000)$ & 98.4 & 95.6 & 97.0 \\
\hline$F A B_{\text {mel }}(15 / 100 / 4000)$ & 98.0 & 95.6 & 96.8 \\
\hline$F A B_{m e l}(20 / 100 / 4000)$ & 98.4 & 95.6 & 97.0 \\
\hline$F A B_{\text {mel }}(30 / 100 / 4000)$ & 97.6 & 95.2 & 96.4 \\
\hline$F A B_{\text {uni }}(10 / 15)$ & 96.0 & 93.2 & 94.6 \\
\hline$F A B_{\text {uni }}(15 / 15)$ & 97.6 & 93.2 & 95.4 \\
\hline$F A B_{\text {uni }}(15 / 20)$ & 96.0 & 93.2 & 94.6 \\
\hline$F A B_{\text {uni }}(20 / 20)$ & 97.2 & 92.8 & 95.0 \\
\hline LEBESGUE (15/15/5000) & 91.6 & 79.2 & 85.4 \\
\hline LEBESGUE (20/20/5000) & 92.0 & 80.4 & 86.2 \\
\hline LEBESGUE $(15 / 15 / 10000)$ & 90.4 & 80.4 & 85.4 \\
\hline LEBESGUE (20/20/10000) & 90.8 & 80.8 & 85.8 \\
\hline LEBESGUE (15/15/15000) & 90.8 & 80.8 & 85.8 \\
\hline LEBESGUE (20/20/15000) & 90.0 & 81.2 & 85.6 \\
\hline
\end{tabular}

with the mel-cepstrum coefficients. Most of the energy of the sound and vibration signals is typically located at the lowfrequency bands. For this reason, the mel-cepstrum coefficients emphasize the low-frequency bands. As such, the frequency amplitude bins matched the filters used in Section 2.2. The frequency spectrum was divided into 15,20 , and 30 frequency bins with the respective boundaries indicated in Tables 1 and 2 . $F A B_{\text {mel }}$ represents the results of the non-uniform mel frequency division results.

The $F A B_{\text {mel }}$ coefficients are computed using Eq. (8). The subband frequency values are given in Table 3 . The subband frequency values produce inferior results compared to the mel-cepstrum coefficients.

In addition to using non-uniform frequency bands as used in mel-cepstrum, uniform frequency division was also performed where the bandwidth of each bin is equal and correspond to $\frac{4000}{2 * 15}$ and $\frac{4000}{2 * 20}$ respectively. The results of uniform frequency band division are represented as $F A B_{u n i}$ in Tables 4 and $5 . F A B_{\text {uni }}(X / Y)$ indicates that the first $X$ frequency bands out of $Y$ frequency bands are used for training and testing.

\subsection{Lebesgue Fourier features}

The feature vectors comprising non-uniform frequency division based on the amplitude levels were also extracted. These features are called Lebesgue features because the Lebesgue integral is based on the division of the vertical axis. In this approach, the integral function of the Fourier transform magnitude is computed and divided into bins according to the amplitude levels, as shown in Figs. 6 and 7. Lebesgue $5000(10,000)$ indicates that the integral of the Fourier transform magnitude is divided into bins below the amplitude level of $5000(10,000)$.

\subsection{Classification}

An SVM using a radial basis function, and a linear kernel was used for the classification process. The radial basis function was used with different gamma parameters. The best performances in 1024 and 2048 sampled datasets are $98.0 \%$, 97.0\%, and $96.2 \%$ for gamma values 1,2 , and 10, respectively. Kernel parameters are not required for the linear kernel because it uses the Euclidean norm. The number of support vectors is 260 for RBF and 255 for the linear kernel, respectively. The SVM software of Libsvm (Chang

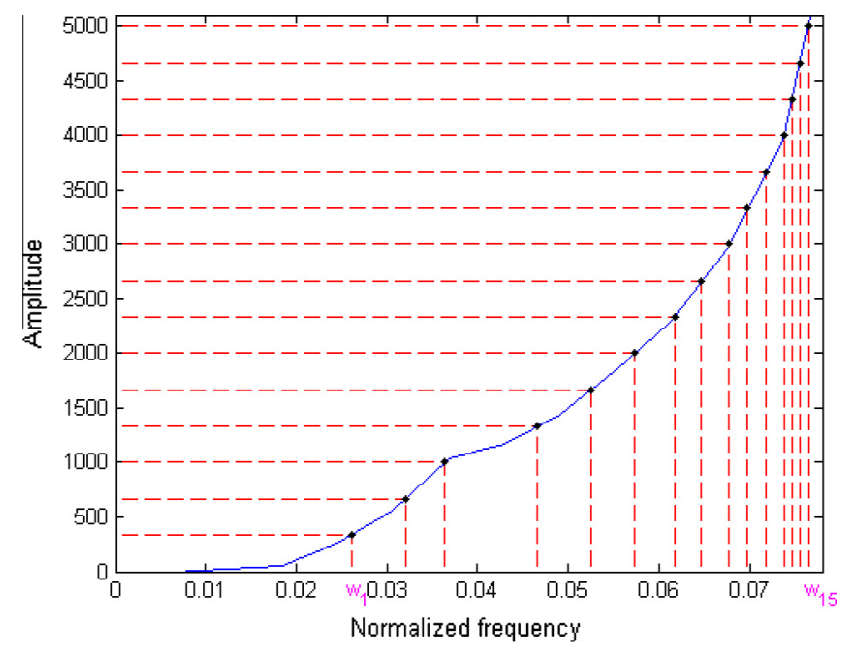

Fig. 6. Lebesgue Fourier features of a nut: Frequency values $w_{1}, \ldots, w_{15}$ are used to form a feature vector. 


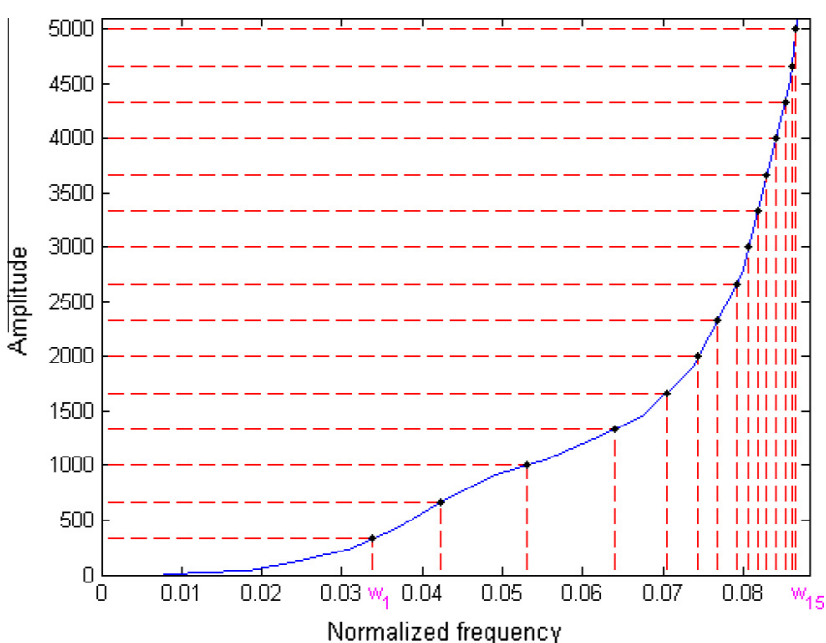

Fig. 7. Lebesgue Fourier Features of a shell piece: Frequency values $w_{1}, \ldots, w_{15}$ are used to form a feature vector.

Table 5

Recognition success rates (sample length is 1024).

\begin{tabular}{|c|c|c|c|}
\hline Feature vector & Shell piece (\%) & Hazelnut (\%) & Average (\%) \\
\hline CHOG (15/15) & 85.2 & 87.2 & 86.2 \\
\hline CHOG $(20 / 20)$ & 88.4 & 89.2 & 88.8 \\
\hline MFCC (15/15) & 96.0 & 97.2 & 96.6 \\
\hline MFCC (20/20) & 96.0 & 97.6 & 96.8 \\
\hline MFCC (10/30) & 95.2 & 98.8 & 97.0 \\
\hline $\operatorname{MFCC}(15 / 30)$ & 96.8 & 98.0 & 97.4 \\
\hline $\operatorname{MFCC}(10 / 50)$ & 95.2 & 98.8 & 97.0 \\
\hline MFCC (15/50) & 96.0 & 98.0 & 97.0 \\
\hline MFCC (10/100) & 95.6 & 98.8 & 97.2 \\
\hline MFCC (15/100) & 96.0 & 98.0 & 97.0 \\
\hline MFCC (20/100) & 96.0 & 97.2 & 96.6 \\
\hline MFCC (30/100) & 95.6 & 96.4 & 96.0 \\
\hline LSF (10/15) & 83.2 & 99.2 & 91.2 \\
\hline LSF (15/15) & 85.2 & 100.0 & 92.6 \\
\hline LSF $(15 / 20)$ & 86.8 & 100.0 & 93.4 \\
\hline LSF $(20 / 20)$ & 86.8 & 99.6 & 93.2 \\
\hline$F A B_{\text {mel }}(15 / 15)$ & 96.0 & 92.8 & 94.4 \\
\hline$F A B_{\text {mel }}(20 / 20)$ & 96.0 & 92.4 & 94.2 \\
\hline$F A B_{\text {mel }}(10 / 30)$ & 96.8 & 94.8 & 95.8 \\
\hline$F A B_{\text {mel }}(15 / 30)$ & 95.2 & 94.8 & 95.0 \\
\hline$F A B_{\text {mel }}(10 / 50)$ & 98.0 & 95.6 & 96.8 \\
\hline$F A B_{\text {mel }}(15 / 50)$ & 97.2 & 94.8 & 96.0 \\
\hline$F A B_{m e l}(10 / 100)$ & 97.6 & 93.6 & 95.6 \\
\hline$F A B_{\text {mel }}(15 / 100)$ & 97.2 & 95.6 & 96.4 \\
\hline$F A B_{\text {mel }}(20 / 100)$ & 98.0 & 96.0 & 97.0 \\
\hline$F A B_{\text {mel }}(30 / 100)$ & 97.2 & 94.4 & 95.8 \\
\hline$F A B_{\text {uni }}(10 / 15)$ & 96.4 & 92.0 & 94.2 \\
\hline$F A B_{\text {uni }}(15 / 15)$ & 96.0 & 91.6 & 93.8 \\
\hline$F A B_{\text {uni }}(15 / 20)$ & 95.6 & 92.0 & 93.8 \\
\hline$F A B_{\text {uni }}(20 / 20)$ & 96.0 & 90.8 & 93.4 \\
\hline LEBESGUE (15/15/5000) & 85.2 & 84.0 & 84.6 \\
\hline LEBESGUE (20/20/5000) & 84.4 & 82.8 & 83.6 \\
\hline LEBESGUE (15/15/10000) & 84.8 & 84.0 & 84.4 \\
\hline LEBESGUE (20/20/10000) & 84.4 & 83.6 & 84.0 \\
\hline LEBESGUE (15/15/15000) & 86.0 & 82.8 & 84.4 \\
\hline LEBESGUE $(20 / 20 / 15000)$ & 84.4 & 83.6 & 84.0 \\
\hline
\end{tabular}

and Lin, 2011) was used in the experimental studies. The experimental results were obtained using vibration recordings of 500 nuts and 500 shell pieces. Some shell pieces and hazelnuts were used several times because shape of some pieces and hazelnuts were not similar to the other ones. This might cause the false detection on the vibration sensor. The SVM was trained using 250 nuts and 250 shell recordings, and the recognition results were obtained using the remaining data.

\section{Results and conclusions}

Table 4 compares the various feature extraction schemes described in Section 2. The best performance was obtained using the mel-cepstral features: $97.2 \%$ of the shell pieces and $99.2 \%$ of the hazelnuts are correctly classified using the SVM with the linear kernel. This performance corresponds to an average recognition rate of $98.2 \%$ when 2048 data samples are used. The recognition rate decreases to $97.4 \%$ when all 1024 samples are used as shown in Table 5.

The system can be implemented in real time. The computational cost of implementing the system is rather low because a 1024- or 2048-point FFT operation is performed only when a nut or a shell piece triggers an alarm. This reduces the computational cost and it is a measure against the unusual vibrations in the environment. If the system computed the FFT of the vibration signal all the time, this would be a burden on the processor. Whenever the laser triggers the system we are sure that the dominant signal is due to a kernel or a shell piece. Extracted MFCC features are fed to a SVM for classification. The inverse DCT calculation and SVM operation are also not computationally intensive.

When 1024 samples are used, it is possible to process more nuts compared to when 2048 samples are used. It is possible to classify 4 nuts/s when 1024 samples are used in this system.

\section{Conclusion}

The ability to remove shell pieces from hazelnut kernels using impact vibration signals was experimentally verified. MFCCs describing the impact vibration signals produce the best classification accuracy in a SVM-based classification engine. MFCCs are the most widely used speech and sound representation parameters. The MFCCs can successfully represent vibration signals as well.

The system is easily trainable; therefore, it can be used to remove shell pieces from other nut kernels, including pistachio nuts and walnuts.

\section{References}

Buerano, J., Zalameda, J., Ruiz, R., 2012. Microphone system optimization for free fall impact acoustic method in detection of rice kernel damage. Comput. Electron. Agric. 85, 140-148.

Cataltepe, Z., Cetin, A.E., Pearson, T., 2004a. Identification of insect damaged wheat kernels using transmittance images. In: 2004 International Conference on Image Processing, 2004. ICIP'04. IEEE, pp. 2917-2920.

Cataltepe, Z., Pearson, T., Cetin, A.E., 2004b. Fast insect damage detection in wheat kernels using transmittance images. In: Proceedings. 2004 IEEE International Joint Conference on Neural Networks, 2004. IEEE, pp. 1343-1346.

Cataltepe, Z., Enis Cetin, A., Pearson, T., 2005. Identification of insect damaged wheat kernels using transmittance images. Electron. Lett. 41, 238-240.

Cetin, A.E., Pearson, T., Tewfik, A., 2004. Classification of closed and open shell pistachio nuts using principal component analysis of impact acoustics. In: Proceedings.(ICASSP'04). IEEE International Conference on Acoustics, Speech, and Signal Processing, 2004. IEEE, pp. V-677.

Cetin, A.E., Pearson, T., Yardimci, Y., Dulek, B., Onaran, I., 2005. Detection of empty hazelnut from fully developed nuts by impact acoustics. In: Proceedings of EUSIPCO.

Chang, C.C., Lin, C.J., 2011. LIBSVM: a library for support vector machines. ACM Trans. Intell. Syst. Technol. 2, 27:1-27:27, http://www.csie.ntu.edu.tw/cjlin/ libsvm.

Chen, Y.C., Hu, M.L., Cheng, C.W., 2011. Applying non-destructive techniques to inspect preserved egg products by decay rates. J. Food Eng. 104, 30-35.

Erzin, E., Cetin, A.E., Yardimci, Y., 1995. Subband analysis for robust speech recognition in the presence of car noise. In: 1995 International Conference on Acoustics, Speech, and Signal Processing, 1995. ICASSP-95. IEEE, pp. 417-420.

Haff, R., Pearson, T., 2007. Separating in-shell pistachio nuts from kernels using impact vibration analysis. Sens. Instrum. Food Qual. Safety 1, 188-192.

Ince, N., Onaran, I., Pearson, T., Tewfik, A., Cetin, A.E., Kalkan, H., Yardimci, Y., 2008. Identification of damaged wheat kernels and cracked-shell hazelnuts with impact acoustics time-frequency patterns. Trans. ASABE 51, 1461-1469.

Michihiro, S., Takahisa, N., 2012. Method for Measuring Crispness of Food Product. WO Patent 2,012,011,494. 
Omid, M., Mahmoudi, A., Omid, M.H., 2010. Development of pistachio sorting system using principal component analysis (PCA) assisted artificial neural network (ANN) of impact acoustics. Expert Syst. Appl. 37, 7205-7212.

Onaran, I., Dulek, B., Pearson, T., Yardimci, Y., Çetin, A.E., 2005. Detection of empty hazelnuts from fully developed nuts by impact acoustics. In: Proceedings of the 13th European Signal Processing Conference (EUSIPCO'05), Citeseer.

Pearson, T., Cetin, A.E., Tewfik, A., 2005. Detection of insect damaged wheat kernels by impact acoustics. In: IEEE International Conference on Acoustics, Speech, and Signal Processing, 2005. Proceedings (ICASSP'05), IEEE, pp. v-649.
Pearson, T., Cetin, A.E., Tewfik, A., Gokmen, V., 2007a. An overview of signal processing for food inspection [applications corner]. Signal Process. Mag., IEEE 24, 106-109. Pearson, T., Cetin, A.E., Tewfik, A., Haff, R., 2007b. Feasibility of impact-acoustic emissions for detection of damaged wheat kernels. Digital Signal Process. 17, 617-633.

Rabiner, L.R., Schafer, R.W., 1979. Digital Processing of Speech Signals, vol. 19. IET. Yorulmaz, O., Pearson, T., Cetin, A.E., 2011. Cepstrum based feature extraction method for fungus detection. In: Proc. of SPIE Vol, pp. 80270E-1.

Yorulmaz, O., Pearson, T., Cetin, A.E., 2012. Detection of fungal damaged popcorn using image property covariance features. Comput. Electron. Agric. 84, 47-52. 